\title{
A NEW TIMING RECOVERY ARCHITECTURE FOR FAST CONVERGENCE
}

\author{
Piya Kovintavewat, John R. Barry \\ Georgia Institute of Technology \\ Atlanta, GA 30332, USA
}

\author{
M. Fatih Erden, Erozan Kurtas \\ Seagate Technology \\ Pittsburgh, PA 15222, USA
}

\begin{abstract}
For any given communication channel, it is desirable to recover all the initial timing information during acquisition and/or any phase and frequency changes during tracking as fast as possible. In this paper, we propose the oversampled per-survivor processing (PSP) timing recovery (TR) architecture to achieve fast convergence rate in the applications of magnetic recording channels. Its performance is compared with the symbol-rate PSP-based TR, the oversampled conventional TR (OCTR), and also with the symbolrate conventional TR (CTR) architecture used in today's magnetic recording read-channel chip architectures. Results indicate that the oversampled PSP (OPSP) TR yields the best bit-error rate (BER) performance among other TR architectures when fast convergence is required.
\end{abstract}

\section{INTRODUCTION}

Timing recovery is a crucial component in a communication system. It is employed to adjust the sampling phase offset used to sample the received signal so that the sampler output will be synchronized with the transmitted symbol. Practically, it is desirable to achieve synchronization as fast as possible. This means that all the initial phase and frequency offsets in the system during acquisition, and any phase and frequency changes during tracking should be recovered very fast (i.e., with less number of samples).

In this paper, we focus on magnetic recording channels and show that the CTR does not perform well if we want to recover all the sampling phase and frequency information very fast, say within 100 samples. This forms a motivation for us to look for other TR architectures. In [1], we have analyzed the OCTR operating at $T_{s}=T / 2$ (where $T_{s}$ is a sampling period and $T$ is a bit period) in magnetic recording channel architectures, and have illustrated that the OCTR provides better performance than the CTR. In this paper, we first briefly explain the TR architecture called the "PSPVA," which is a PSP-based [2] TR implemented based on a Viterbi algorithm (VA) [3]. Then, we combine the oversampled timing recovery method with the PSP-VA to obtain the oversampled PSP-VA (OPSP-VA) TR architecture, which has the advantages of both the PSP-VA and the oversampled TR architectures, and propose it for fast convergence of synchronization.

\section{SYSTEM MODEL}

Fig. 1 shows a system model for magnetic recording channels. A binary input sequence $a_{k} \in\{ \pm 1\}$ with bit pe- riod $T$ is filtered by an ideal differentiator $1-D$ to form a transition sequence $b_{k} \in\{-2,0,2\}$, where $b_{k}= \pm 2$ corresponds to a positive or a negative transition and $b_{k}=0$ corresponds to the absence of a transition. The transition response, $g(t)$, for longitudinal recording is taken as $g(t)$ $=1 /\left(1+\left(2 t / P W_{50}\right)^{2}\right)$ where $P W_{50}$ determines the width of $g(t)$ at half of its peak value, whereas that for perpendicular recording is $g(t)=\operatorname{erf}\left(2 t \sqrt{\ln 2} / P W_{50}\right)$ where $\operatorname{erf}(\cdot)$ is an error function and $P W_{50}$ indicates the width of the derivative of $g(t)$ at half its maximum. In the context of magnetic recording, a normalized recording density is defined as ND $=P W_{50} / T$, which determines how many data bits can be packed within the resolution unit $P W_{50}$. The media jitter noise, $\Delta t_{k}$, is modeled as a random shift in the "transition position" with a Gaussian probability distribution function with zero mean and variance $\left|b_{k} / 2\right| \cdot \sigma_{j}^{2}$ (i.e., $\left.\Delta t_{k} \sim \mathcal{N}\left(0,\left|b_{k} / 2\right| \cdot \sigma_{j}^{2}\right)\right)$ truncated to $T / 2$, where $|x|$ takes the absolute value of $x$. The clock jitter noise, $\tau_{k}$, is modeled as a random walk according to $\tau_{k+1}=\tau_{k}+\mathcal{N}\left(0, \sigma_{w}^{2}\right)$.

The readback signal, $p(t)$, can then be expressed as

$$
\begin{aligned}
p(t)= & \sum_{k=-\infty}^{\infty} a_{k}\left\{g\left(t-k T-\Delta t_{k}-\tau_{k}\right)\right. \\
& \left.-g\left(t-(k+1) T-\Delta t_{k+1}-\tau_{k}\right)\right\}+n(t),
\end{aligned}
$$

where $n(t)$ is additive white Gaussian noise (AWGN) with power spectral density $N_{0} / 2$. The readback signal $p(t)$ is filtered by a seventh-order Butterworth low-pass filter whose cutoff frequency is at $N /(2 T)$ and then sampled at $t_{m}=m T_{s}+\hat{\tau}_{k}$, where $T_{s}=T / N, N \in\{1,2\}$ is an oversampling ratio, and $\hat{\tau}_{k}$ is the sampling phase offset at time $k$ ( $k=\lfloor m / N\rfloor$ where $\lfloor\cdot\rfloor$ takes on the smallest integer value). The $T / N$-spaced received sequence, $r_{m}$, is equalized by a $T / N$-spaced equalizer, $F(D),(N=1$ corresponds to a $T$ spaced equalizer (TSE), whereas $N=2$ corresponds to a fractionally-spaced equalizer (FSE)) and then downsampled to obtain a $T$-spaced sequence, $y_{k}$, (i.e., $y_{k}=x_{N k}$ ) closely resembling a desired sequence, $d_{k}$. Note that the design of a generalized partial response (GPR) target, $H(D)$, and its corresponding equalizer, $F(D)$, can be found in [4]. Hence, a timing error detector (TED) utilizes $x_{m}$ and $\hat{d}_{k}$ to generate the estimated timing error, $\hat{\epsilon}_{k}$. Note that a symbol detector used for CTR and OCTR in this paper is a Viterbi detector (VD) with a decision delay of $4 T$.

For the symbol-rate system (i.e., $N=1$ ), we considered the Mueller and Müller TED [5], which is given by

$$
\hat{\epsilon}_{k}=x\left(k T+\hat{\tau}_{k}\right) \cdot \hat{d}_{k-1}-x\left((k-1) T+\hat{\tau}_{k-1}\right) \cdot \hat{d}_{k},
$$




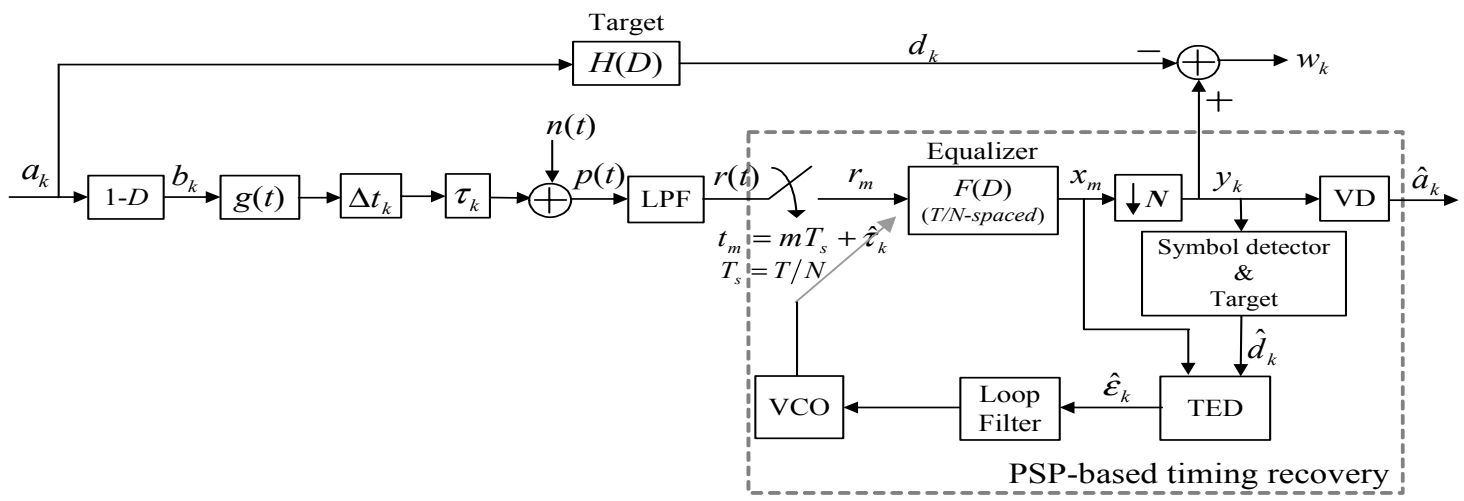

Figure 1: System model with target design.

and for the oversampled system (i.e., $N=2$ ), we picked the early-late TED [6], which is expressed as

$$
\hat{\epsilon}_{k}=\hat{d}_{k} \cdot\left\{x\left(k T+\frac{T}{2}+\hat{\tau}_{k}\right)-x\left(k T-\frac{T}{2}+\hat{\tau}_{k-1}\right)\right\} .
$$

The sampling phase offset is updated by a second-order phase-locked loop (PLL) according to

$$
\begin{aligned}
\hat{\theta}_{k} & =\hat{\theta}_{k-1}+\beta \hat{\epsilon}_{k} \\
\hat{\tau}_{k+1} & =\hat{\tau}_{k}+\alpha \hat{\epsilon}_{k}+\hat{\theta}_{k},
\end{aligned}
$$

where $\theta_{k}$ represents the frequency error, and $\alpha$ and $\beta$ are PLL gain parameters. The PLL gain parameters determine the loop bandwidth and the convergence rate. The smaller the values of $\alpha$ and $\beta$, the smaller the loop bandwidth, the less the noise allowed to perturb the system, and thus the slower the convergence rate. Accordingly, one needs to trade-off between the loop bandwidth and the convergence rate when designing $\alpha$ and $\beta$. Eventually, the VD performs Viterbi detection. Note that the entire block within the dashed box in Fig. 1 represents the PSP-based TR.

\section{PSP-BASED TIMING RECOVERY}

Per-survivor processing (PSP) [2] is a technique for jointly estimating a data sequence and an unknown parameter, e.g., a sampling phase offset. It needs to operate on the trellis structure so that it can exploit the information associated with each state transition (or branch) in the trellis to estimate another unknown parameter. With the PSP technique, we arrive at the (symbol-rate) PSP-VA, which is the PSP-based TR architecture implemented based on a VA. Fig. 2 depicts the trellis structure based on a PR-IV channel $\left(H(D)=1-D^{2}\right)$, which also explains how the PSP-VA works. There are $Q=4$ states labeled as state $(a)$ to state $(d)$, i.e., $Q=\{a, b, c, d\}$. Denote $(p, q)$ as a transition from state $(p)$ to state $(q)$, where $\{p, q\} \in Q$.

Let $\mathbf{r}_{i}^{(p, q)_{k}}$ denote a collection of the sampler outputs, $r_{m}$, at the sampling time index $i$ obtained from the survivor path leading to $(p, q)$ at time $k$. For example, as shown in Fig. 2 with $N=1, \mathbf{r}_{k}^{(b, c)_{k}}=r_{k}^{(b, c)}=r\left(k T+\hat{\tau}_{k}^{(b)}\right)$. Similarly, $\mathbf{r}_{k-1}^{(b, c)_{k}}=r_{k-1}^{(a, b)}=r\left((k-1) T+\hat{\tau}_{k-1}^{(a)}\right)$. Let an $M$-tap

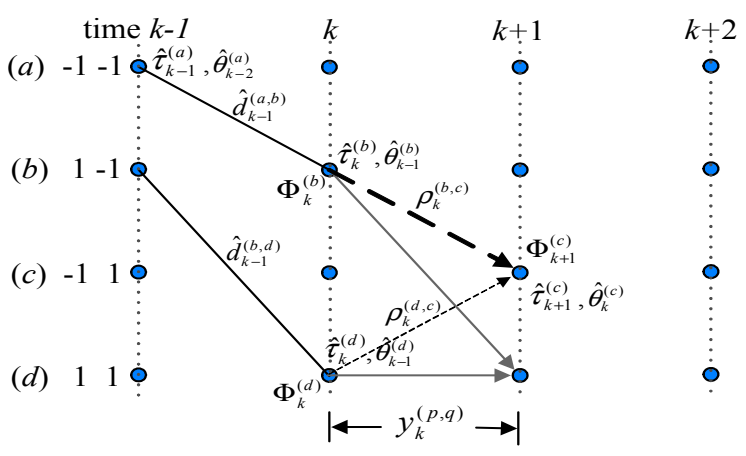

Figure 2: Trellis structure for the PSP-VA.

$(M=2 K+1) T / N$-spaced equalizer take the form $F(D)$ $=\sum_{i=-K}^{K} f_{i} D^{i}$. The equalizer output at symbol interval (called an observation) associated with $(p, q)$ at time $k$ can therefore be expressed as

$$
y_{k}^{(p, q)}=\sum_{i=-K}^{K} f_{i} \mathbf{r}_{N k-K-i}^{(p, q)_{k}} .
$$

Note that $y_{k+\frac{K}{N}}$ will correspond to $a_{k}$. The branch metric associated with $(p, q)$ at time $k$ is defined as

$$
\rho_{k}^{(p, q)}=\left|y_{k}^{(p, q)}-\hat{d}_{k}^{(p, q)}\right|^{2}
$$

where $\hat{d}_{k}^{(p, q)}$ is the $k$-th channel output associated with $(p, q)$. Suppose there are two branches, $(u, q)$ and $(p, q)$, arriving at state $(q)$ time $k+1$, where $u \in Q$. The path metric at state $(q)$ time $k+1$ is then defined as

$$
\Phi_{k+1}^{(q)}=\min \left\{\Phi_{k}^{(u)}+\rho_{k}^{(u, q)}, \Phi_{k}^{(p)}+\rho_{k}^{(p, q)}\right\} .
$$

A transition leading to a minimum path metric $\Phi_{k+1}^{(q)}$ will be considered as a survivor path up to state $(q)$ time $k+1$.

The key idea of the PSP-VA is to sample the received signal using different sampling phase offsets associated with each branch. Additionally, each state in the trellis has 
its own PLL to update the sampling phase offset, whereas each branch contains one analog-to-digital converter and one equalizer. The PSP-VA algorithm is similar to the VA, except an additional timing update operation. Note that associated with each survivor path there are a path metric, a channel output, an observation, a frequency error, and a sampling phase offset. To achieve fast convergence rate, we take advantages of the PSP-VA and the oversampled TR studied in [1] to realize the OPSP-VA.

\subsection{The Oversampled PSP-VA}

The OPSP-VA performs in the same way as the PSP-VA does, except that the received signal $r(t)$ is sampled at $T_{s}$ $=T / 2$ and an FSE is used. Note that an amount of $K / 2$ will represent the delay in bit period. For simplicity, we assume that $K$ is divisible by 2 . Let $L$ be the length of $a_{k}$, i.e., $\boldsymbol{a}=\left[a_{0}, a_{1}, \ldots, a_{L-1}\right]$. Then, the OPSP-VA algorithm can be summarized as follows:

The OPSP-VA algorithm:

1. Initialize $\hat{\boldsymbol{\tau}}_{0}^{(q)}=0$ for all $q \in Q$.

2. For $k=0,1,2, \ldots, L-1+(K / 2) \quad\left(\right.$ set $a_{k}=0$ for $\left.k \geq L\right)$

For $q=a, b, c, d$

- Consider two transitions arriving at state $(q)$ at time $k+1$, e.g., $(u, q)$ and $(p, q)$ where $\{u, p\} \in Q$.

- Use $\hat{\tau}_{k}^{(u)}=\hat{\tau}_{k}^{(p)}=0$ if $k<K / 2$.

- Sample $r(t)$ at $T_{s}=T / 2$ using $\hat{\tau}_{k}^{(u)}$ and $\hat{\tau}_{k}^{(p)}$ to obtain $\left[r_{2 k}^{(u, q)}, r_{2 k+1}^{(u, q)}\right]$ and $\left[r_{2 k}^{(p, q)}, r_{2 k+1}^{(p, q)}\right]$.

- Equalize the sampler outputs to obtain $\left[x_{2 k}^{(u, q)}, x_{2 k+1}^{(u, q)}\right]$ and $\left[x_{2 k}^{(p, q)}, x_{2 k+1}^{(p, q)}\right]$.

- Obtain two observations by taking $y_{k}^{(u, q)}=x_{2 k}^{(u, q)}$ and $y_{k}^{(p, q)}=x_{2 k}^{(p, q)}$.

- Compute two branch metrics $\rho_{k}^{(u, q)}$ and $\rho_{k}^{(p, q)}$.

- Choose a transition leading to a minimum path metric at state $(q)$ time $k+1$.

- Suppose a transition $(p, q)$ is chosen to be a survivor path, then compute $\hat{\epsilon}_{k}^{(p, q)}$ using (3).

- Update $\hat{\theta}_{k}^{(q)}$ and $\hat{\tau}_{k+1}^{(q)}$ based on $\hat{\epsilon}_{k}^{(p, q)}, \hat{\theta}_{k-1}^{(p)}$ and $\hat{\tau}_{k}^{(p)}$.

- Update the path metric $\Phi_{k+1}^{(q)}$.

End

End

3. $\hat{\boldsymbol{\tau}}$ and $\hat{\mathbf{a}}$ are obtained from the survivor path that has the minimum path metric. Note that only $\hat{\tau}_{i}$ and $\hat{a}_{i}$ for $i=$ $(K / 2),(K / 2)+1, \cdots, L-1+(K / 2)$ will correspond to the input sequence due to the delay introduced by an FSE.

\section{SIMULATION RESULTS}

We consider $\mathrm{ND}=2.5$ for both longitudinal and perpendicular recording channels with $\sigma_{j} / T=3 \%$ media jitter noise, $\sigma_{w} / T=0.5 \%$ clock jitter noise, and $0.4 \%$ frequency offset. The SNR is defined as SNR $=10 \log _{10}\left(V_{p}^{2} / \sigma^{2}\right)$ in dB, where $V_{p}$ is the peak amplitude of the isolated transition (assumed to be 1) and $\sigma^{2}=N_{0} /\left(2 T_{s}\right)$ is the input AWGN power. The 5 -tap GPR target and a 21-tap equalizer were designed at the $\mathrm{SNR}$ required to achieve $\mathrm{BER}=10^{-5}$. We use a linearized model of PLL [7] to design $\alpha$ and $\beta$ assuming that there is no noise in the system and the S-curve slope [7] is
Table I: PLL gain parameters for longitudinal recording.

\begin{tabular}{cccccc}
\hline Convergence & \multicolumn{4}{c}{ Timing recovery schemes } \\
\cline { 2 - 6 } rate & CTR & PSP-VA & OCTR & OPSP-VA \\
(in bit periods) & $D=14 T$ & $D=10 T$ & $D=9 T$ & $D=5 T$ \\
\hline \hline$C=256$ & $\alpha$ & 0.0027 & 0.0028 & 0.0043 & 0.0045 \\
\cline { 2 - 6 }$C$ & $\beta$ & $3.11 \mathrm{e}-5$ & $3.24 \mathrm{e}-5$ & $5.22 \mathrm{e}-5$ & $5.44 \mathrm{e}-5$ \\
\hline$C=100$ & $\alpha$ & 0.0057 & 0.0062 & 0.0098 & 0.0107 \\
\cline { 2 - 6 } & $\beta$ & $1.43 \mathrm{e}-4$ & $1.63 \mathrm{e}-4$ & $2.65 \mathrm{e}-4$ & $3.09 \mathrm{e}-4$ \\
\hline$C=50$ & $\alpha$ & 0.0087 & 0.0098 & 0.0158 & 0.0189 \\
\cline { 2 - 6 } & $\beta$ & $3.91 \mathrm{e}-4$ & $4.74 \mathrm{e}-4$ & $7.71 \mathrm{e}-4$ & $9.88 \mathrm{e}-4$ \\
\hline
\end{tabular}

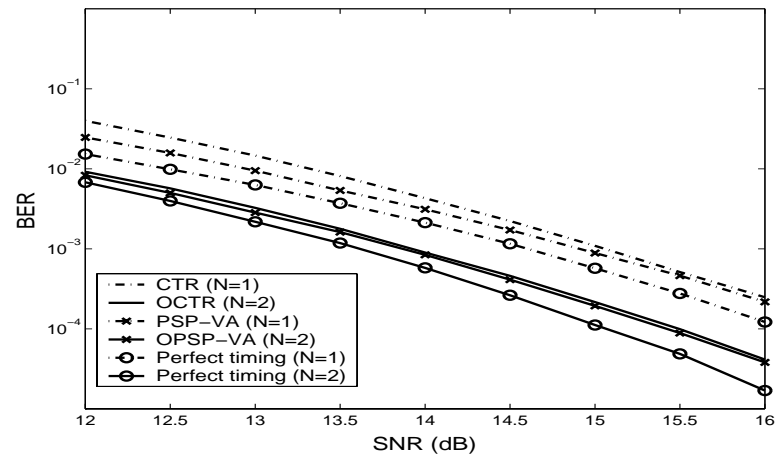

Figure 3: BER performance of different TR schemes for longitudinal recording using $\alpha$ and $\beta$ designed for $C=256$.

equal to one at the origin. The PLL gain parameters were designed to recover phase and frequency changes in $C$ bit periods (the smaller the $C$, the faster the convergence rate). Note that the PLL gain parameters highly depend on the chosen target, a total delay (denoted as $D$ ) in the timing loop, a given $C$, and a TED algorithm. For a given $D, \alpha$ is designed to recover phase change in $C$ bit periods with $\pm 5 \%$ tolerance. Upon having $D$ and $\alpha, \beta$ is then designed to recover $0.4 \%$ frequency offset within $C$ bit periods. The same PLL gain parameters are used during acquisition and tracking modes.

The proposed TR architecture (i.e., the OPSP-VA) will be compared with the PSP-VA, the CTR, and the OCTR. Note that each TR scheme experiences different loop delays. It is easy to show that the total loop delays of CTR, OCTR, PSP-VA and OPSP-VA are 14T, 9T, $10 T$ and 5T, respectively (as TSE, FSE and the symbol detector introduce delays of $10 T, 5 T$ and $4 T$, respectively). Finally, each BER point was computed using as many data packets as needed to collect at least 1000 error bits. One data packet consists of $C$-bit preamble ( $4 T$ pattern) and a 4096 -bit input data sequence.

\subsection{Longitudinal Recording}

The 5-tap GPR target for the symbol-rate system is $H(D)$ $=1+0.613 D-0.478 D^{2}-0.626 D^{3}-0.291 D^{4}$, whereas that for the oversampled system is $H(D)=1+0.419 D-$ $0.441 D^{2}-0.544 D^{3}-0.268 D^{4}$. The PLL gain parameters for different TR schemes are shown in Table I. Fig. 3 shows BER performance of different TR schemes using $\alpha$ and $\beta$ designed for $C=256$. The curve labeled "Perfect timing" means that the receiver uses $\hat{\tau}_{k}=\tau_{k}$ to sample the received signal. With perfect synchronization, the oversampled sys- 

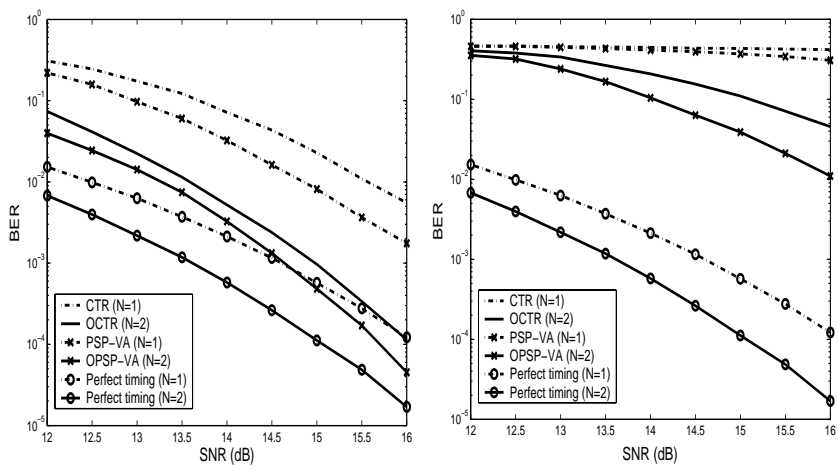

Figure 4: BER performance of different TR schemes for longitudinal recording using $\alpha$ and $\beta$ designed for $C=100$ (left) and $C=50$ (right).

tem itself offers a large performance gain over the symbolrate system. This suggests that the oversampled system should be employed in a longitudinal recording channel.

As depicted in Fig. 3, for a given TR architecture (PSPbased or conventional), the oversampled system outperforms the symbol-rate system for all cases. However, for a given system (oversampled or symbol-rate), the PSP-based TR is just slightly better than the conventional one. This is because they operate at "the optimal point", where $\alpha$ and $\beta$ were designed to minimize the steady-state error in the timing loop (based on a linearized model), regardless of the convergence rate. The $\alpha$ and $\beta$ designed for $C=256$ can be given as an example. Nevertheless, the performance gain gets large when employing $\alpha$ and $\beta$ designed for a small $C$ (i.e., when operating in a system that requires a fast convergence rate) as illustrated in Fig. 4. Clearly, the OPSP-VA yields the best performance among other TR schemes for all cases. This also implies that the OPSP-VA can achieve a faster convergence rate than any other TR scheme.

\subsection{Perpendicular Recording}

The 5-tap GPR target for the symbol-rate system is $H(D)$ $=1+1.429 D+1.097 D^{2}+0.465 D^{3}+0.099 D^{4}$, whereas that for the oversampled system is $H(D)=1+1.421 D+$ $1.076 D^{2}+0.451 D^{3}+0.097 D^{4}$. The PLL gain parameters for different TR schemes are shown in Table II. Unlike longitudinal recording, we observed that there is no significant performance gain between the oversampled system and the symbol-rate system in perpendicular recording when operating a system with $\alpha$ and $\beta$ designed for $C=256$. However, a relatively large gain can be obtained between the oversampled system and the symbol-rate system, and between the PSP-based TR architecture and the conventional TR architecture when employing $\alpha$ and $\beta$ designed for $C=$ 100 and $C=50$, as depicted in Fig. 5. Again, the OPSP-VA performs better than other TR schemes for all cases.

\section{CONCLUSION}

In this paper, we proposed a new $\mathrm{TR}$ architecture called the OPSP-VA to achieve a fast convergence rate. We have shown that the OPSP-VA performs better than other TR
Table II: PLL gain parameters for perpendicular recording.

\begin{tabular}{cccccc}
\hline Convergence & \multicolumn{4}{c}{ Timing recovery schemes } \\
\cline { 2 - 6 } rate & CTR & PSP-VA & OCTR & OPSP-VA \\
(in bit periods) & $D=14 T$ & $D=10 T$ & $D=9 T$ & $D=5 T$ \\
\hline \hline$C=100$ & $\alpha$ & 0.0070 & 0.0076 & 0.0129 & 0.0140 \\
\cline { 2 - 6 }$C=5$ & $\beta$ & $1.76 \mathrm{e}-4$ & $2.02 \mathrm{e}-4$ & $3.48 \mathrm{e}-4$ & $4.06 \mathrm{e}-4$ \\
\hline$C=50$ & $\alpha$ & 0.0107 & 0.0121 & 0.0207 & 0.0248 \\
\cline { 2 - 6 } & $\beta$ & $4.83 \mathrm{e}-4$ & $5.86 \mathrm{e}-4$ & $1.01 \mathrm{e}-3$ & $1.30 \mathrm{e}-3$ \\
\hline
\end{tabular}
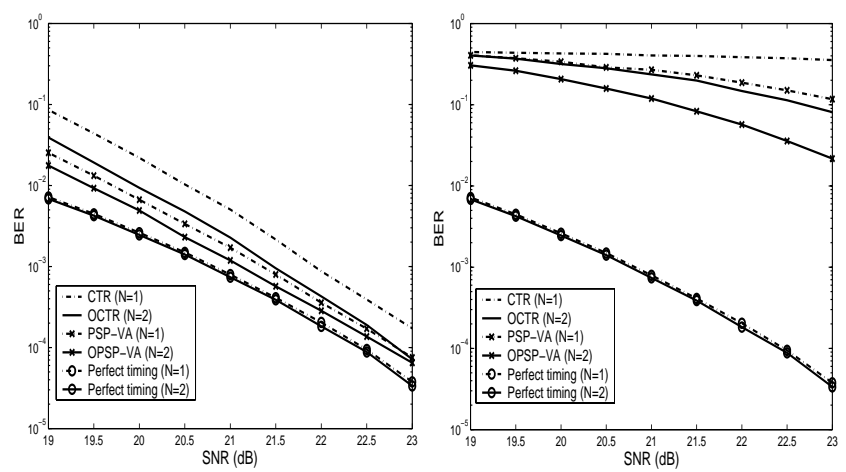

Figure 5: BER performance of different TR schemes for perpendicular recording using $\alpha$ and $\beta$ designed for $C=$ 100 (left) and $C=50$ (right).

schemes, especially when operating in a system that requires a fast convergence rate.

As the complexity of the oversampled TR architecture [1] and the PSP-based [2] TR architecture is high, all advantages gained by the OPSP-VA need to be balanced against the increased implementation cost.

\section{REFERENCES}

[1] P. Kovintavewat, M. F. Erden, E. Kurtas, and J. R. Barry, "Oversampled timing recovery for magnetic recording channels," submitted to IEEE Trans. on Magnetics, September 2003.

[2] R. Raheli, A. Polydoros, and Ching-Kae Tzou, "The principle of per-survivor processing: a general approach to approximate and adaptive MLSE," IEEE Globecom'91, vol. 2, pp. 1170-1175.

[3] G. D. Forney, "Maximum-likelihood sequence estimation of digital sequences in the presence of intersymbol interference," IEEE Trans. on Information Theory, vol. IT-18, no. 3, pp. 363-378, May 1972.

[4] J. Moon and W. Zeng, "Equalization for maximum likelihood detector," IEEE Trans. on Magnetics, vol. 31, no. 2, pp. 1083-1088, March 1995.

[5] K. Mueller and M. Müller, "Timing recovery in digital synchronous data receivers," IEEE Trans. on Communications, 24:516-531, May 1976.

[6] P. Mallory, "A maximum likelihood bit synchronizer," International Telemetring Conf., Proc., IV (1968), pp. 1-16.

[7] J. W. M. Bergmans, Digital baseband transmission and recording. Boston/London/Dordrecht: Kluwer Academic Publishers, 1996. 